\title{
Diabetes tipo MODY; presentación en un lactante
}

\author{
MC. Cubero Sobrados ${ }^{a}$, O. Martín Gordo ${ }^{b}$, D. Agüero Orgaz ${ }^{b}$

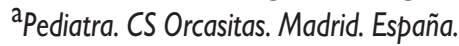 \\ ${ }^{b}$ Médico de Familia. CS Orcasitas. Madrid. España.
}

Fecha de publicación en Internet: 20 de septiembre de 2011

\section{Resumen}

La diabetes tipo MODY (del inglés Maturity Onset Diabetes of the Young) se caracteriza porque los enfermos debutan con menos de 25 años, porque su herencia es autosómica dominante (por eso es frecuente encontrar miembros de tres generaciones de una misma familia afectados) y porque no necesitan insulina al menos en los cinco años posteriores al diagnóstico. Se conocen siete genes implicados en la diabetes MODY. La mayoría de ellos son factores de transcripción que intervienen en el desarrollo embrionario. Según cuál sea el gen implicado se puede clasificar en distintos tipos, siendo MODY 2 y MODY 3 los más comunes. Se presenta un caso de diabetes tipo MODY en un niño de ocho meses de edad.

Palabras clave: Diabetes tipo MODY. Hiperglucemia. Insulinorresistencia.

Type MODY diabetes; presentation in an infant

Abstract

The diabetes type MODY (of the English Maturity Onset Diabetes of the Young) is characterized by the patients starting when they are less than 25 years old, due to the fact that its inheritance is autosomal dominant (for that reason it is frequent to find 3 generations affected in a same family) and by not needing insulin at least in the 5 years following the diagnosis. Seven implied genes are known for diabetes MODY. The majority of them are transcription factors that take part in the embryonic development. According to what gene is the implied one they can be classified in different types. The most common are MODY 2 and MODY 3. A case of diabetes MODY in a boy 8 months old is presented.

Key words: Type MODY diabetes. Hyperglycemia. Insulin-resistance.

\section{Introducción}

La diabetes de comportamiento del adulto que se presenta en el joven, denominada MODY (del inglés Maturity
Onset Diabetes of the Young), es un subtipo heterogéneo de diabetes mellitus tipo 2 que se presenta por una alteración genética caracterizada por tener



Los autores declaran no presentar conflictos de intereses en relación con la preparación y publicación de este artículo. 
un carácter autosómico dominante y presentar una secreción defectuosa de insulina sin insulinorresistencia. En el presente artículo revisamos la presentación de diabetes tipo MODY en un lactante de ocho meses.

\section{Caso clínico}

Lactante de ocho meses que acude al centro de salud por presentar escasa ganancia ponderal durante el último mes, motivo por el cual se realizó un examen de orina en el que se objetiva glucosuria (300 mg/dl), y una glucemia capilar de $127 \mathrm{mg} / \mathrm{dl}$ (dos horas después de la merienda). No refiere poliuria, polidipsia, vómitos, fiebre, ni otra sintomatología. Se repiten controles de glucemia capilar posprandiales resultando altos, por lo que se remite a un centro hospitalario para estudio.

\section{Antecedentes personales}

Embarazo controlado, diabetes mellitus gestacional diagnosticada en el tercer trimestre y controlada con dieta. Parto eutócico. Recién nacido pretérmino (RNPT) $(35+5 \mathrm{~s})$ con peso al nacimiento de $2260 \mathrm{~g}$ y longitud de $49 \mathrm{~cm}$. Hiperbilirrubinemia neonatal. Lactancia materna exclusiva cuatro meses, después lactancia mixta. Al nacimiento: anoniquia en los dos dedos índices y en la uña del dedo medio de la mano izquierda con ausencia parcial. Inmunización completa para su edad. Profilaxis contra raquitismo con vitamina $D_{3}$. Sin alergias conocidas hasta la fecha.

\section{Antecedentes familiares}

Madre de 37 años, sana; G3-A1-V2.

Diabetes mellitus gestacional en el anterior embarazo; menarquia a los nueve años; talla $165 \mathrm{~cm}$. Padre de 34 años. Glucemias basales altas (100-125 $\mathrm{mg} / \mathrm{dl}$ ), sin sobrepeso; talla $175 \mathrm{~cm}$. Hermanastro (vía paterna) de 12 años, RNPT de peso elevado para la edad gestacional. Abuela materna hipertensa. Abuela paterna diabética en tratamiento con dieta. Historia familiar importante de hiperglucemia en la rama paterna: abuela, tres tíos-abuelos, tres tíos y dos primos paternos.

\section{Pruebas complementarias}

- Hemograma: hemoglobina: 12,5 $\mathrm{g} / \mathrm{dl}$; plaquetas: 698 000/ $\mathrm{mm}^{3}$; leucocitos: $16700 / \mathrm{mm}^{3}$ (neutrófilos: $24 \%$, linfocitos: $65,9 \%$ ).

- Perfil hepatorrenal: normal; glucosa: $120 \mathrm{mg} / \mathrm{dl}$; sodio: $142 \mathrm{mEq} /$; potasio: $4 \mathrm{mEq} / \mathrm{dl}$.

- Gasometría venosa: ph: 7,45; bicarbonato: $23,2 \mathrm{mmol} / \mathrm{l} ; \mathrm{pCO}_{2}$ : $32,8 \mathrm{~mm} \mathrm{Hg}$. 
- Sistemático de orina: ph: 6,5; densidad: 1020; resto normal. Sedimento normal.

- Insulina: inferior a $2 \mathrm{mU} / \mathrm{l}$, péptido C: $0,49 \mathrm{ng} / \mathrm{ml}$ (valores normales 0,5-2,0 ng/ml); hemoglobina glucosilada $5,1 \%$. Hormonas tiroideas normales. Anticuerpos de enfermedad celiaca negativos. Perfil lipídico normal. Estudio de autoinmunidad de diabetes mellitus: anti-GAD65, A-antiinsulina, anti-IA2 negativos e inmunoglobulina $D$ : $1 \mathrm{mg} / \mathrm{dl}$.

\section{Exploración física}

Peso de $6500 \mathrm{~g}(-2 /-3$ desviaciones estándar [DS]); talla de $67 \mathrm{~cm} \mathrm{(-1/-2}$ DS). Buen estado general, bien hidratado y perfundido; auscultación cardiopulmonar normal; abdomen normal. Estadio Tanner I (T1-P1-A1); testes de $2 \mathrm{ml}$ de Prader en bolsas. Ausencia de uña en los dedos índices de ambas manos y ausencia parcial de uña del tercer dedo de la mano izquierda. Exploración neurológica y osteoarticular normal.

\section{Evolución}

El paciente en su evolución presentó controles de glucemia capilar que oscilaron entre 100 y $183 \mathrm{mg} / \mathrm{dl}$, mante- niendo en todo momento un excelente estado general. No presentó glucosuria, cetonuria ni poliuria-polidipsia en ningún momento. No ha precisado tratamiento alguno.

\section{Discusión}

Dentro de la diabetes tipo MODY se han identificado varios subtipos ${ }^{1-6}$; aunque su prevalencia es poco conocida, se estima que la forma más común es el tipo $2(63 \%)^{4}$, que presenta mutaciones en el gen de la glucocinasa ${ }^{1,2,4}$. Las manifestaciones clínicas son poco agresivas, no se asocia a obesidad, hipertensión ni alteraciones lipídicas. El fallo secretor de las células beta se puede evidenciar por la falta del aumento del péptido $C$ tras un estímulo ${ }^{1,4,6}$. Le sigue en frecuencia el MODY tipo $3^{4}$, asociado a defectos en el factor nuclear hepático 1-alfa en el que se han identificado 30 mutaciones diferentes, asocia una forma grave de diabetes mellitus postpuberal, con retinopatía, proteinuria y que requiere tratamiento insulínico. El MODY $1^{1,2,4,5}$, menos frecuente, originado por mutaciones en el gen del factor nuclear hepático 4-alfa, causa una diabetes grave con desarrollo frecuente de complicaciones. Hay identificadas otras formas más raras y poco frecuentes, MODY 4, 5 y 6 , y se sugiere la existen- 
cia de otros defectos genéticos desconocidos hasta el momento y que se han dado en denominar MODY $X^{4,5}$, describiéndose en 2006 en dos familias el MODY 7 con afectación pancreática'.

A menudo es difícil el diagnóstico del gen implicado, ya que los factores de transcripción afectados en este tipo de diabetes pueden estar interrelacionados. Aun así, cuando sea posible, el análisis genético facilita un diagnóstico precoz que permite estudiar a estos pacientes y sus familiares y poder prevenir complicaciones agudas y crónicas asociadas a esta patología, como pueden ser importantes complicaciones microo macrovasculares y llevar a cabo un pronóstico dependiente del factor genético, haciendo posible un tratamiento específico.

Normalmente, el estudio completo de estos pacientes se lleva a cabo en el ámbito hospitalario, donde es posible el acceso a pruebas especializadas y genéticas. El diagnóstico genético es importante, pues permite individualizar el tipo de trata- miento ${ }^{6}$, ningún tratamiento en los casos MODY 2, dosis bajas de sulfonilureas en los MODY 1 o 3,2,6. Desde Atención Primaria nos corresponde, como en este caso, hacer un diagnóstico diferencial temprano en el estudio de lactantes o niños con fallo de medro o, en el extremo opuesto, niños o jóvenes con sobrepeso u obesidad en los casos de presentación temprana de diabetes no monogénicas, y en todos ellos tener muy presentes los antecedentes tanto familiares como personales ${ }^{3}$.

La importancia del diagnóstico precoz radica en evitar tratamientos crónicos e innecesarios, clasificar correctamente a ambos progenitores y poder llevar a cabo un seguimiento clínico y analítico de estos pacientes y sus descendientes. La historia natural o evolución va a depender del gen o genes afectados $y$, aunque inicialmente se ha tratado de asociar un tipo de diabetes a una clínica y evolución, lo más importante es el seguimiento clínico y analítico personalizado del paciente.

\section{Bibliografía}

1. Barrio R. Diabetes monogénicas: enfoque diagnóstico y tipos más frecuentes. Av Diabetol. 2007;23(5):333-40.
2. Gómez-Ayala AE. Diabetes tipo MODY: La diabetes del adulto en la etapa infanto-juvenil. Medwave. 2010;X(2).

3. Tapia Ceballos L, Córdoba Borras E, Picazo Angelín B, Ranchal Pérez P. Diabetes MODY. 
Una causa frecuente de hiperglucemia. An Pediatr (Barc). 2008;69:565-7.

4. Carmena R. Complejidad de la diabetes mellitus tipo 2. En: Segovia de Arana JM, Mora Teruel F. Ochoa y la medicina clínica. Madrid: Farmaindustria; serie Científica; 2004. p. 79-88.
5. Sánchez-Reyes L, Fanghänel G. Actualización en los diferentes subtipos de diabetes tipo "MODY". Rev Endocrinol Nutr. 2001:9(1):5-11.

6. Barrio R. Diabetes tipo 2 en la edad pediátrica. Pediatr Integral. 2003;VII(7):504-11. 